\title{
Simulation numérique du creusement d'une galerie dans un massif argileux gonflant
}

\section{J.-C. ROBINET}

\section{S. TACHERIFET}

Euro-Géomat Consulting

51, route d'Olivet

45100 Orléans

Tél. : 38.66.22.20

Fax: 38.66 .24 .70

Centre de Recherche sur la Matière divisée, Groupe Géomatériaux, CNRS-ESEM rue Léonard-de-Vinci 45072 Orléans cedex 2

Tél. : 38.51 .78 .79

Fax: 38.63 .37 .96
L'article présente une modélisation du comportement mécanique des argiles gonflantes saturées. Cette modélisation est associée à un modèle de comportement qui tient compte du gonflement élastoplastique de l'argile, en utilisant deux surfaces de charges, et sans couplage avec le fluide interstitiel. Le modèle permet de décrire les chemins contrainte-déformation du matériau à l'état normalement consolidé et surconsolidé. Le calcul est effectué à long terme par la prise en compte des caractéristiques drainées du matériau. Ainsi les chemins de décharge dans le massif, provoqués par l'excavation. sont décrits en contrainte totale.

La modélisation est validée dans un premier temps par des essais de laboratoire, odométrique et triaxial réalisés sur la bentonite et sur l'argile de Boom. La comparaison des résultats mesurés et des résultats calculés montre que l'ensemble des phénomènes sont décrits.

La modélisation est appliquée ensuite à la simulation du creusement d'une galerie circulaire réalisée dans une argile gonflante peu perméable. On montre que la décompression du massif suite à l'excavation se traduit par une plastification du massif proche de la paroi et par une chute des contraintes dans la zone plastique. Cette diminution de contraintes entraîne une forte convergence de la galerie qui atteint quelques centimètres en paroi. Les résultats numériques sont comparés avec les solutions analytiques proposées par Rousset (1988), et montrent que le modèle développé interprète de façon satisfaisante le comportement de la galerie après la décharge.

\section{Numerical simulation of excavation of gallery in expansive clay}

The paper presents a modelling of mechanical behaviour of saturated expansive clays. This modelling is based on behaviour model including elastoplastic swelling of clay by using two plastic mechanisms. The study was carried out without association of interstitial fluid. The calculation was performed in drained conditions. The unloading paths in rock mass induced by the excavation was expressed in term of total stress. The modelling is fitted first on laboratory tests performed on bentonite and Boom clay, the comparison between plotted and measured results shows that all the phenomena are described. Then the modelling was applied to simulate the excavation of gallery realised in expansive, impermeable clayey mass (Boom clay). We show that the decompression after excavation induces a plastification around the underground and a decrease of stresses in plastic zone. This decrease of stresses provoke the high convergence of gallery. The numerical results were compared with analytical solution proposed by Rousset (1988) and show that the modelling interpret correctly the mechanical behaviour of tunnel. 
niques

$\gamma_{w} \quad$; poids volumique de l'eau

$\gamma_{h}$ : poids volumique humide

$\sigma_{\mathrm{x}} \quad$ : contrainte horizontale

$\sigma_{y} \quad$ : contrainte verticale

$\sigma_{x y} \quad$ : contrainte tangentielle

$\sigma_{-} \quad$ : contrainte axiale

$\mathrm{K}_{0} \quad$ : coefficient de poussée des terres au repos

$\mathrm{u}_{0} \quad$ : pression interstitielle initiale

$\sigma_{n} \quad$ : contrainte normale

$\sigma_{n}^{\prime} \quad$ : contrainte normale effective

$\sigma_{\mathrm{n}} \quad$ : composante horizontale de la contrainte normale

$\mathrm{O}_{\mathrm{n}, \mathrm{r}} \quad$ : composante verticale de la contrainte normale angle d'inclinaison de la facette par rapport à l'horizontale

$\mathrm{u}_{x} \quad$ : déplacement horizontal

$\mathrm{u}_{\mathrm{y}} \quad$ : déplacement vertical

Ui : convergence

$\mathrm{f}_{1} \quad$ : vecteur forces volumiques

$T_{1} \quad$ : vecteur forces de surface

$\sigma_{i \|} \quad$ : tenseur des contraintes

$\varepsilon_{\|} \quad$ : tenseur des déformations

$u_{i} \quad$ : vecteur des déplacements

$\mathrm{C}_{\mathrm{j} k \mathrm{k})}$ : matrice d'élasticité

$\Delta \sigma_{\| 1}^{0} \quad$ : tenseur de correction des contraintes

K ; matrice de rigidité globale

$\Delta u \quad$ : vecteur des déplacements nodaux

$\Delta F \quad$ : vecteur des forces extérieures

\section{1 \\ Introduction}

La décharge mécanique d'un sol argileux gonflant saturé, lors de la réalisation des fondations de bâtiments, des tunnels ou des fouilles est à l'origine de nombreux désordres dans les structures de Génie civil dus à un gonflement important. Ce gonflement du sol engendre des soulèvements de bâtiments (David et al., 1980) et donne naissance à des efforts parasites, qui provoquent des frottements négatifs dans les pieux (Mohan et Chandra, 1961; Prakash et al., 1987; Sorochan; 1989), des cisaillements aux points de liaison des radiers et des semelles filantes, et un accroissement des poussées sur le soutènement des galeries.

Ainsi depuis plusieurs années, les constructeurs ont mis en cuvre des techniques de construction pour limiter les causes de gonflement (Chen, 1988; Mouroux et al., 1988).

Les études entreprises dans ce domaine se limitent bien souvent à l'étude du phénomène en laboratoire, à travers des essais de gonflement odométrique Didier et Soyez (1987). Par ailleurs, les calculs analytiques élastiques de gonflement par l'utilisation des paramètres déterminés au laboratoire sous-estiment les déplacements réels de l'ouvrage. L'absence de modèles rhéologiques prenant en compte le gonflement ne permet pas de quantifier correctement ces déplacements dans les argiles gonflantes.

La réalisation d'essais de laboratoire sur le comportement des argiles gonflantes, par exemple l'argile de Boom et la smectite montre que ces argiles présentent des caractéristiques spécifiques, en particulier une augmentation de la pente de décharge avec la pression de consolidation, et des déformations volumiques plastiques sous un cycle de décharge-recharge (Baldi et al., 1990).

Habituellement dans les argiles, les déformations plastiques provoquées par la consolidation sont décrites par la plasticité. En revanche, les déformations de gonflement liées aux contraintes de répulsion lors 
de la décharge mécanique sont souvent traduites par l'élasticité et ne tient pas compte des déformations plastiques de gonflement. La seule connaissance de la pression de gonflement n'est pas suffisante, et ces déformations plastiques nécessitent le développement d'un modèle de comportement approprié.

Ainsi un modèle élastoplastique a été développé pour décrire le comportement des argiles gonflantes (Robinet et Pakzad, 1994), que nous avons introduit dans un code de calcul par éléments finis. Ce modèle élastoplastique comporte deux mécanismes plastiques :

- le premier mécanisme, associé à un écrouissage isotrope est représenté par le modèle Cam-Clay pour décrire le comportement normalement consolidé (phase de chargement):

- le deuxième mécanisme à plasticité non associative, à écrouissage combiné isotrope et cinématique, permet de décrire le gonflement élastoplastique en décharge (état surconsolidé).

La modélisation numérique de l'excavation d'une galerie creusée à une grande profondeur dans un massif argileux gonflant a été effectuée avec ce modèle, dans le cadre d'une thèse de doctorat (Tacherifet, 1995).

Après une analyse physique du gonflement dans les argiles saturées, une brève description du modèle élastoplastique prenant en compte le gonflement en décharge dans les argiles sera présentée. Le calage du modèle a été réalisé sur des essais homogènes (essais triaxiaux sur l'argile de Boom (Baldi et al., 1990), essai cyclique odométrique sur une bentonite MX-80 (Böorgesson et al., 1993)). La validation de la modélisation (problème aux limites) a été étudiée à travers des essais de laboratoire. Le premier calcul concerne un essai odométrique en décharge. Le deuxième calcul traite de la simulation d'un essai cyclique triaxial. Enfin la modélisation est appliquée à la simulation de l'excavation d'une galerie profonde construite dans l'argile de Boom. Les résultats sont comparés d'une part aux solutions analytiques proposées par Rousset (1988) et, d'autre part, aux résultats de calcul obtenus avec le modèle Cam-Clay.

2

\section{Analyse du gonflement dans les argiles}

Le phénomène de gonflement qui apparaît lors de la décharge mécanique d'un échantillon d'arqile saturée est la superposition:

- d'un gonflement cristallin qui concerne les interactions à courte distance, ce mécanisme est commun à toutes les argiles. Il désigne les phénomènes mis en jeu lors de l'hydratation du minéral sec. Il est dû à la fixation des molécules d'eau dans les espaces interlamellaires qui se structurent en couches successives ;

- d'un gonflement osmotique qui concerne des interactions électrostatiques entre les doubles couches ayant lieu pour des dimensions d'espaces poreux plus grandes. Ce deuxième mécanisme est lié à la présence de cations chargés qui se fixent sur les feuillets. Les substitutions ioniques confèrent au feuillet argileux une charge négative, qui est compensée par des cations situés en surface. En présence d'eau, ces cations ont tendance à diffuser vers la solution sous l'effet d'un gradient de concentration en cations, entre les surfaces des feuillets argileux et le reste de la solution en générant une pression osmotique. Israelachvili (1985), Van Damme et al. (1987). Ainsi Lambe (1958) propose d'expliquer le gonflement par l'intermédiaire de la théorie de la double couche diffuse.

De manière générale le gonflement des sols argileux est observé dans deux cas :

i) par décompression d'un sol saturé et ii) par sorption d'un sol à l'état sec alimenté en eau. Nous nous limitons ici à l'étude des phénomènes de gonflement Iorsque le sol gonflant saturé est soumis à une décharge mécanique. En résumé, pour que le phénomène de gonflement dans une argile saturée se produise, les deux conditions suivantes sont nécessaires:

- présence de minéraux argileux gonflants tels que la smectite et la montmorillonite;

- décharge mécanique (état surconsolidé).

Pour décrire le comportement contrainte-déformation dans les argiles gonflantes, Mitchell (1976) et Sridharan (1990) ont étendu le concept des contraintes effectives, en écrivant que la contrainte totale appliquée est la contribution: des contraintes de contact, des pressions interstitielles, et des contraintes d'attraction et de répulsion. Ils proposent la relation suivante:

$$
\sigma=\sigma_{c}+u_{w}+\sigma_{\mathrm{A}-\mathrm{A}}
$$

Nous avons étudié, à partir des essais cycliques odométriques, le gonflement de quatre types d'argiles (argile du Bassin parisien, kaolinite, argile de Boom et smectite). Nous avons réalisé une série d'essais odométriques pour déterminer les caractéristiques mécaniques de ces argiles (Fig. 1). L'analyse des résultats de ces essais montre que:

- les pentes de consolidation à l'état normalement consolidé sont identiques pour les différents matériaux;

- les pentes de décharge sont plus fortes pour les argiles de type Boom et smectite;

- les boucles d'hystérésis après une décharge à $5 \mathrm{MPa}$ sont plus aplaties pour la kaolinite et l'argile du Bassin parisien.

Par conséquent, les argiles de type kaolinite et du Bassin parisien peuvent être classées comme des argiles non gonflantes, par contre la smectite et l'argile de Boom seront classées comme des argiles gonflantes.

Pour les argiles non gonflantes la décharge mécanique de l'échantillon saturé entraîne la diminution des contraintes de contact et une faible augmentation de volume. En revanche, pour les argiles gonflantes, la diminution de la contrainte mécanique entraine une augmentation de la distance moyenne entre les feuillets (du fait de la pression osmotique). En effet, les charges électriques à la surface des feuillets donnent naissance à des forces de répulsion non équilibrées par la force extérieure, qui sont à l'origine de déformations macroscopiques du matériau, Les déformations continuent à se développer jusqu'à ce qu'un nouvel état d'équilibre soit atteint.

\section{3}

\section{Formulation du modèle}

Le modèle élastoplastique pour les argiles gonflantes (Robinet et al., 1994; Pakzad, 1995) peut être décrit de façon simple, il est composé de: 


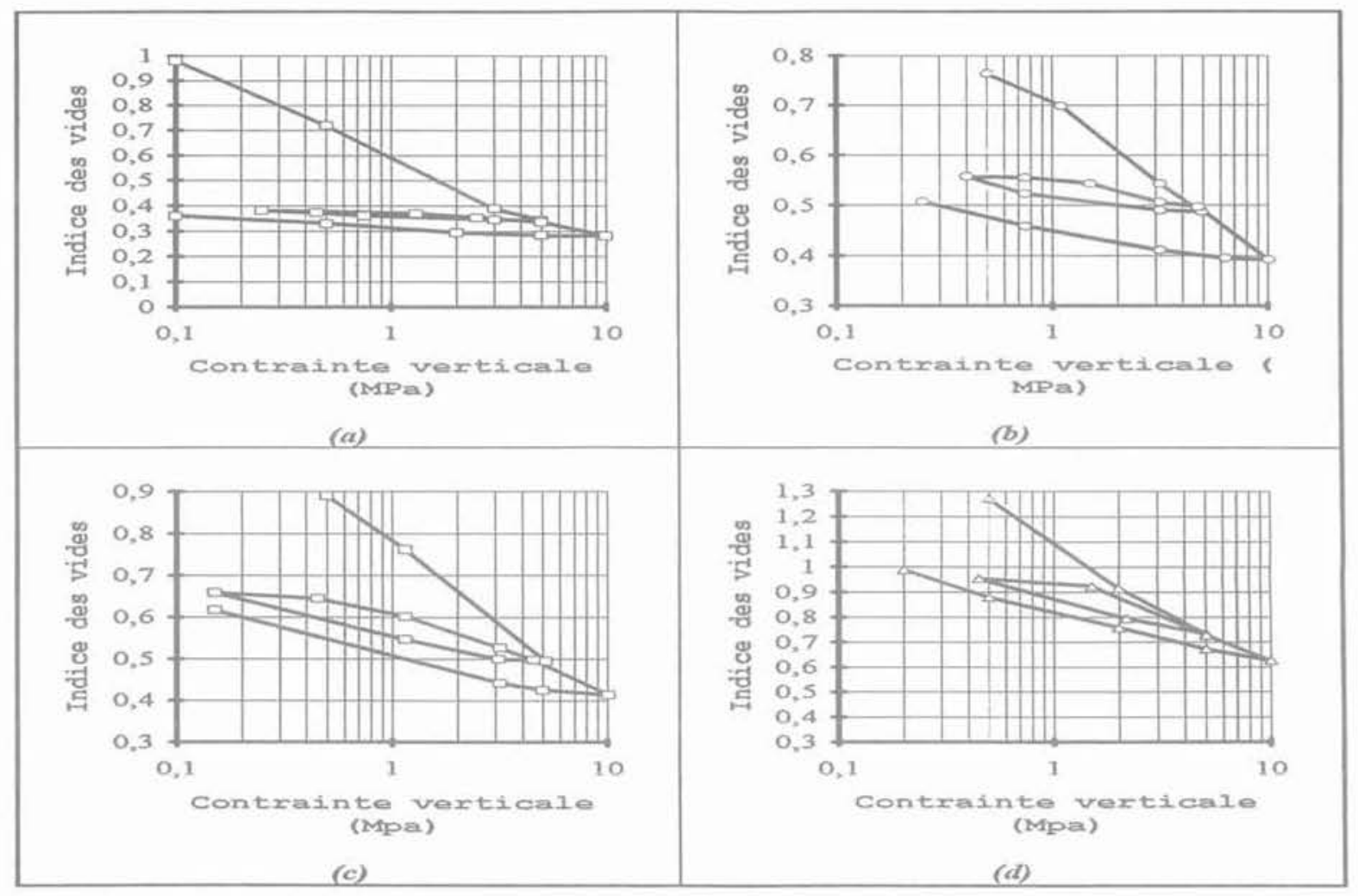

nG.1 Essais odométriques cycliques sur des argiles remaniées saturées (Tacherifet, 1995). "Comparaison du comportement entre argiles gonflantes et non gonflantes ». (a) Argile du Bassin parisien. (b) Kaolinite. (c) Argile de Boom. (d) Argile foca. Cyclic oedometer tests on remoulded saturated clays (Tacherifet, 1995). "Comparison of behaviour between swelling and unswelling clays.

(a) Bassin parisien clay (b) Kaolinite. (c) Boom clay. (d) Foca clay.

- deux surfaces de charge, l'une de Cam-Clay et l'autre de gonflement; elles sont définies en fonction des paramètres d'état critique et d'état limite $\left(M, \beta_{1}, \beta_{2^{\prime}} P_{c \gamma^{\prime}} \alpha_{p^{\prime}}\right.$ $\left.\alpha_{\mathrm{q}^{\prime}} \mathrm{a}_{0,}\left(\mathrm{p}_{\mathrm{c}}\right)_{\text {ref }}\right)$;

- la surface de charge de gonflement associé à un écoulement plastique (loi non associée).

La formulation complète du modèle est décrite dans l'annexe I.

- Simulation des essais homogènes

La simulation numérique de l'essai odométrique cyclique, réalisé par Börgesson et al. (1993) sur la ben- tonite $\mathrm{MX}-80$, est réalisée en utilisant les paramètres rhéologiques suivants :

$\left(\mathrm{p}_{\mathrm{c}}\right)_{\text {ref }}=10 \mathrm{MPa} ; \mathrm{n}=1 ; \mathrm{a}_{0}=0,35 \mathrm{MPa} ; \mathrm{p}_{\mathrm{co}}=14,6 \mathrm{MPa}$ $\beta_{1}=10 ; M=1 ; E_{0}=100 \mathrm{MPa} ; v=0,35$

Les figures $2 \mathrm{a}$ et $2 \mathrm{~b}$ représentent les contraintes calculées et celles mesurées dans le plan (e-logo). La comparaison des résultats montre que le processus de décharge recharge est assez bien simulé par le modèle de gonflement. Par ailleurs le calcul montre une faible hystérésis pour la contrainte horizontale (e - logo ) et une hystérésis assez large pour la contrainte verticale $\left(e-\log \sigma_{y}\right)$.
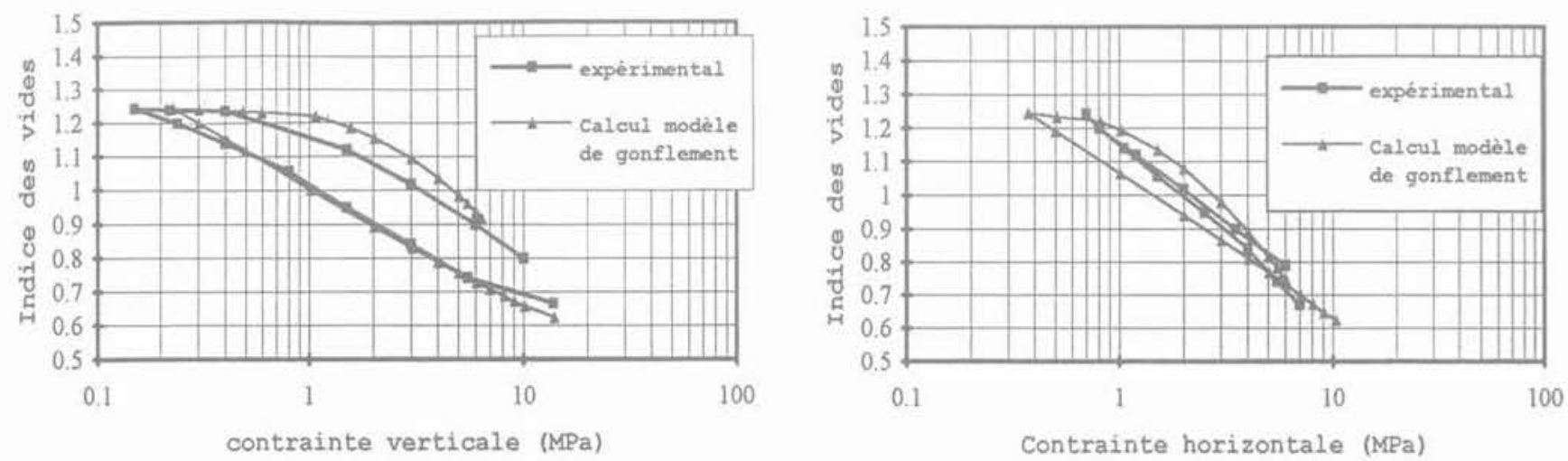

FG. 2. Simulation de l'essai œdométrique cyclique sur la bentonite MX-80. (a) Contrainte verticale $\sigma_{y}$;

(b) Contrainte horizontale $\sigma$.

Simulation of cyclic oedometer test on bentonite MX-80. (a) Vertical stress $\sigma_{y}$ (b) Horizontal stress $\sigma_{x}$ 


\section{Intégration du modèle dans le code éléments finis}

Le modèle de comportement présenté précédemment est introduit dans un code de calcul par éléments finis, afin de simuler le comportement des ouyrages, lors de la décharge mécanique (tel que les fondations, les barrières ouvragées et les galeries souterraines). La formulation variationnelle du problème, puis sa discrétisation conduisent à un système non linéaire avec comme inconnues les déplacements aux nœuds (Arnexe II).

- Résolution numérique du problème

L'algorithme numérique utilisé pour résoudre ce problème d'élastoplasticité est basé sur la méthode des forces résiduelles (méthode de la rigidité initiale). Cette dernière consiste à n'effectuer le calcul de la matrice de rigidité qu'à la première itération du premier incrément de chargement. La solution est obtenue par une succession de résolution de système linéaire, en vêrifiant les conditions de plasticité pour chaque incrément de chargement, et en évaluant pour chaque itération de calcul un résidu mécanique. La solution est supposée atteinte, dès que ce résidu est inférieur à une tolérance fixée.

\section{5}

\section{Validation de la modélisation}

Compte tenu des difficultés numériques rencontrées pour introduire le modèle rhéologique, nous avons procédé à la validation de la modélisation en plusieurs étapes.

Dans une première étape nous avons essayé de reproduire des chemins de décharge et de recharge homogènes: odométrique et triaxiaux. Ceci nous a permis de mieux contrôler le bon déroulement des différentes opérations effectuées dans le programme, à savoir : la résolution du système, le calcul des déformations et des contraintes par retour sur la surface limite, et la compensation des résidus. La deuxième étape de la validation concerne la simulation du creusement des galeries dans un massif argileux gonflant. Cette étape a permis de vérifier, d'une part, le bon déroulement des calculs élastoplastiques pour chaque point qui présente un chemin de décharge particulier et, d'autre part, le fonctionnement du schéma numérique de résolution.

\section{1}

\section{Simulation d'un essai de gonflement oedométrique}

Dans le cas de la simulation du creusement d'une galerie souterraine, de nombreux éléments se trouvent dans la configuration d'une décharge cedométrique. Ce dernier chemin présente donc un intérêt particulier dans une démarche de validation. L'utilisation des modèles classiques ne donne pas une bonne représentation du comportement. En effet, la simulation de la décharge œdométrique avec le modèle Cam-Clay par exemple, conduit à un chemin élastique linéaire avec une pente faible, qui se situe loin du chemin expérimental et les déformations sont sous-estimées.
L'essai oedométrique, réalisé sur un échantillon de bentonite MX-80 (Börgesson, 1993) de $5 \mathrm{~cm}$ de diamètre et $2 \mathrm{~cm}$ de hauteur, est modélisé par un maillage constitué de 9 éléments en utilisant les conditions aux limites suivantes : déplacements à la base de l'échantillon et sur les côtés latéraux sont nuls (Fig. 3). Le module d'Young est considéré variable, compte tenu de la variation de la pression moyenne lors de la décharge. La matrice de rigidité de la structure est recalculée à chaque incrément de déchargement. Le calcul est effectué en utilisant les paramètres mécaniques suivants :

- contraintes initiales

- indice des vides initial

$$
\sigma_{\mathrm{x}}=7 \mathrm{MPa} ; \sigma_{\mathrm{y}}=14 \mathrm{MPa} ; \mathrm{K}_{0}=0,5
$$

$$
e_{0}=0,67
$$

- paramètres rhéologiques

$$
\begin{gathered}
\left(\mathrm{p}_{\text {cref }}=10 \mathrm{MPa} ; \mathrm{n}=1 ; \mathrm{a}_{0}=0,35 \mathrm{MPa} ; \mathrm{p}_{\mathrm{co}}=8,23 \mathrm{MPa}\right. \\
\beta_{1}=10 ; \mathrm{M}=1 ; \mathrm{E}_{0}=100 \mathrm{MPa} ; \mathrm{v}=0,35
\end{gathered}
$$

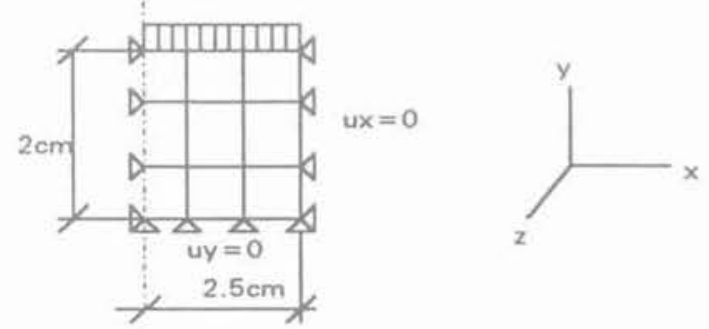

FG. 3 Maillage et conditions aux limites utilisées pour la simulation d'un essai de décharge oedométrique. Mesh and boundary conditions used for simulation of unloading oedometer test.

La décharge s'effectue dans le sens vertical par application de 28 incréments ( $\left.\mathrm{d} \sigma_{\mathrm{y}}=0,5 \mathrm{MPa}\right)$. La figure 4 montre schématiquement l'évolution de la surface de charge de gonflement pendant la décharge dans un essai odométrique. La figure 5 présente les résultats de la simulation dans le plan (e - logo). La comparaison des résultats calculés et des résultats mesurés montre que le processus de décharge est globalement bien simulé pour des contraintes fortes $\left(\sigma_{y}>1 \mathrm{MPa}\right)$.

En revanche, le calcul surestime légèrement les déformations totales dans le domaine des faibles contraintes $\left(\sigma_{i}>0,6 \mathrm{MPa}\right)$. Le gonflement de l'échantillon à la fin de la décharge atteint $0,7 \mathrm{~cm}$ (pour une hauteur initiale de l'échantillon de $2 \mathrm{~cm}$ ) sous une contrainte finale de $0,3 \mathrm{MPa}$.

\section{2}

\section{Simulation d'un essai triaxial}

On simule dans ce deuxième exemple un essai triaxial cyclique légèrement surconsolidé réalisé sur l'argile de Boom (Baldi et al., 1990). 


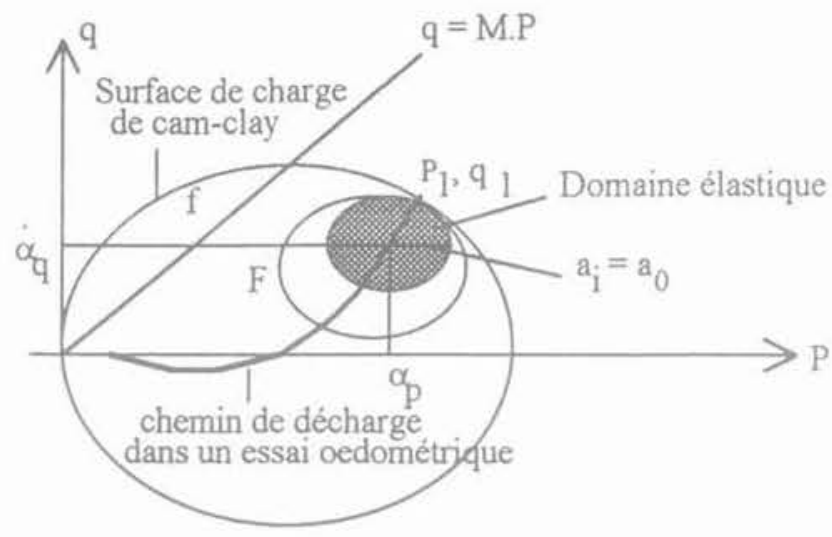

FG.4 Schếmatisation de l'évolution de la surface de charge du modèle de gonflement en décharge cedométrique.

Schematic evolution of yield surface « $\mathrm{F}$ i of swelling model during unloading in oedometer test.

Etant donné la symétrie du problème, uniquement le quart de l'échantillon a été étudié. Un maillage de 9 éléments et de 16 nceuds est utilisé pour modéliser un échantillon de $6,5 \mathrm{~cm}$ de diamètre et $6,5 \mathrm{~cm}$ de hauteur (Fig. 6).

- Contraintes initiales

$$
\sigma_{\mathrm{x}}=\sigma_{\mathrm{y}}=\sigma_{\mathrm{z}}=4 \mathrm{MPa}
$$

- Paramètres rhéologiques

$$
\begin{gathered}
p_{t 0}=6 \mathrm{MPa} ; a_{0}=0,6 \mathrm{MPa} ; \mathrm{E}_{0}=500 \mathrm{MPa} \\
\beta_{1}=18 ;\left(p_{)_{\text {ref }}}=10 \mathrm{MPa} ; v=0,35\right. \\
\mathrm{M}=0,83 ; \mathrm{n}=1
\end{gathered}
$$

La figure 7 présente les résultats de la simulation. La comparaison entre les résultats expérimentaux et les résultats prédits par le calcul montre que l'ensemble des phénomènes sont décrits, en particulier les déformations volumiques de dilatance en décharge, et les déformations volumiques de contractance en recharge à l'état surconsolidé. La boucle d'hystérésis est assez bien modélisée. Celle-ci devient plus large lorsque la contrainte de décharge augmente.
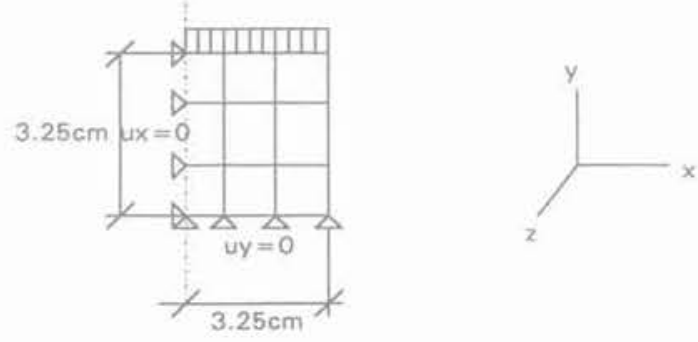

FG. 6 Maillage de l'échantillon et conditions aux limites. Mesh and boundary conditions of sample.

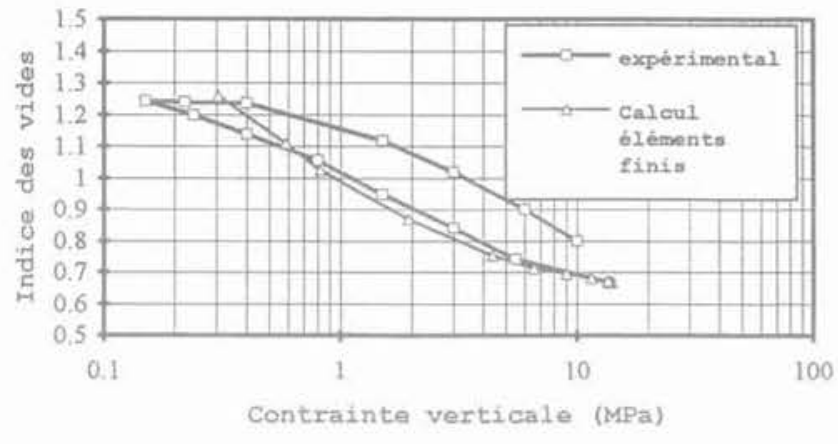

fG. S Simulation numérique d'un essai de décharge oedométrique réalisé sur la bentonite MX-80, calcul avec le code Géoméca.

Numerical simulation of unloading oedometer test on bentonite MX-80 calculation with Géoméca code.

\section{Simulation du creusement d'une galerie circulaire dans un massif argileux}

La galerie circulaire d'un diamètre de $6 \mathrm{~m}$ est creusée à $250 \mathrm{~m}$ de profondeur dans l'argile de Boom. La modélisation numérique traite en déformations planes une section verticale du massif. Le domaine modélisé possède un axe de symétrie oy, de ce fait la moitié de la galerie est étudiée. Les conditions aux limites de la structure sont: déplacements horizontaux nuls au niveau de l'axe de la galerie en raison de la symétrie, déplacements horizontaux et verticaux nuls aux limites du maillage (points qui se trouvent suffisamment loin de l'excavation). La structure est constituée de 72 éléments isoparamétriques à quatre nœuds Q4. Le maillage avec les conditions aux limites est représenté sur la figure 8. L'excavation est modélisée par une décharge incrémentale jusqu'à l'obtention d'une contrainte normale nulle sur les parois.

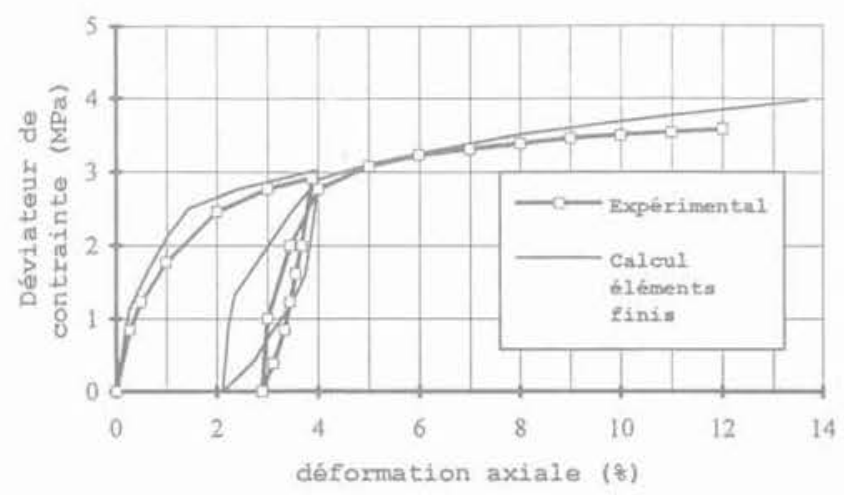

FIG.7 Simulation numérique de l'essai triaxial cyclique drainé « argile de Boom normalement consolidée $n$. $\mathrm{OCR}=1,5$, contrainte de confinement $=4$ $\mathrm{MPa}$; calcul avec le code Géoméca. Numerical simulation of cyclic drained triaxial test $\alpha$ Bloom clay, normally consolidated $z$ $O C R=1.5$; confinement pressure $=4 \mathrm{MPa}$, calculation with Géoméca code. 
Le contour circulaire est modélisé par un ensemble de facettes inclinées chacune d'un angle $\beta$ par rapport à l'horizontale.

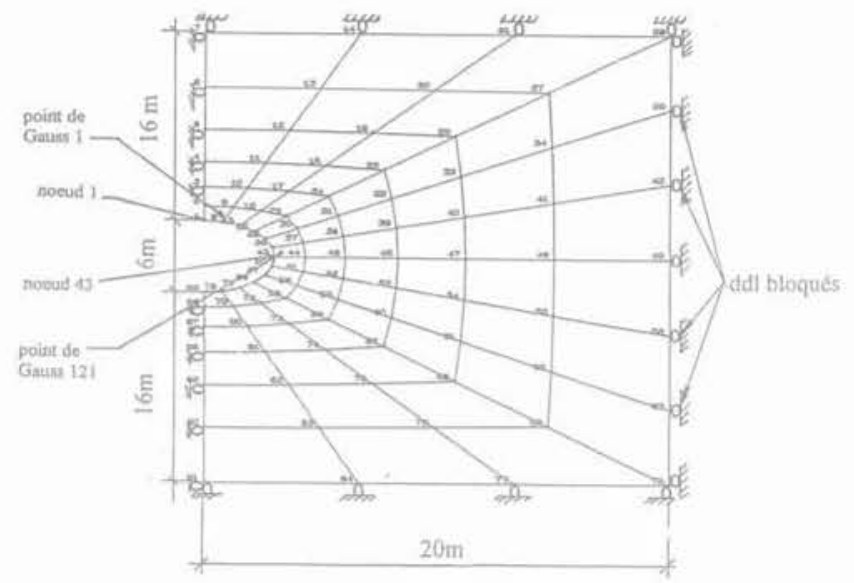

FG, 8 Maillage et conditions aux limites d'une galerie circulaire.

Mesh and boundary conditions of circular galiery.

Le vecteur contrainte normale qui s'exerce sur une facette inclinée d'un angle $\beta$ est donnée par:

avec:

$$
\sigma_{n}=\sigma_{n}^{\prime}+u_{0}
$$

$\mathrm{u}_{0}=\gamma_{\mathrm{w}}, \mathrm{z}=2,5 \mathrm{MPa}$

$\sigma_{Y}=4,98 \mathrm{MPa} ; \sigma_{X}=4,48 \mathrm{MPa} ; K_{0}=\frac{\sigma_{X}^{\prime}}{\sigma_{y}^{\prime}}=0,8$

où:

$\sigma_{\text {v }}^{\prime}$ est la contrainte effective verticale;

$\mathrm{K}_{0}$ est le coefficient de poussée des terres au repos;

$\beta$ est l'angle d'inclinaison de la facette.

Les valeurs des contraintes horizontale et verticale in situ, qui s'exercent à $250 \mathrm{~m}$ de profondeur pour deux poids volumiques, sont données dans le tableau I.
Les composantes horizontales et verticales des contraintes totales à appliquer sur le contour correspondant aux différentes facettes sont résumées dans le tableau II $\left(\sigma_{n x}=\sigma_{n} \cdot \sin (\beta) ; \sigma_{n y}=\sigma n \cdot \cos (\beta)\right)$.

Le problème ainsi posé concerne le calcul des contraintes et des déplacements au sein du massif ayant un comportement élastoplastique, suite à l'application de ces contraintes de déconfinement sur les facettes. Les modélisations numériques ont été conduites avec le modèle de Cam-Clay et le modèle de gonflement. Les résultats sont comparés à des solutions analytiques dans le cas d'un massif élastique et élastoplastique (critère de Mohr-Coulomb).

\section{Caration}

\section{Caractéristiques mécaniques du calcul}

- Contraintes initiales

$u_{0}=2,5 \mathrm{MPa}$

$$
\sigma_{\mathrm{Y}}=4,98 \mathrm{MPa} ; \sigma_{\mathrm{X}}=4,48 \mathrm{MPa} ; \mathrm{K}_{0}=\frac{\sigma_{x}^{\prime}}{\sigma_{y}^{\prime}}=0,8
$$

Paramètres rhéologiques

$$
\left(\mathrm{p}_{\mathrm{c}}\right)_{\text {ref }}=10 \mathrm{MPa} ; \mathrm{a}_{0}=0,3 \mathrm{MPa} ; \mathrm{p}_{\mathrm{ce}}=5 \mathrm{MPa} ; \beta 1=18 \text {; }
$$$$
\mathrm{M}=0,86 ; \mathrm{E}_{0}=500 \mathrm{MPa} ; \mathrm{v}=0,35 \text {. }
$$

\section{- Tolérances}

- sur la surface limite : flim $=0,01$

-sur le résidu: $\quad$ : $\quad 0,05$.

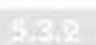

\section{Analyse des résultats de la simulation numérique}

\section{a) Contraintes dans le massif}

L'excavation de la galerie engendre une chute des contraintes radiales et des contraintes axiales dans la zone plastique qui se développe autour de l'excavation. Une augmentation des contraintes orthoradiales, tant

\begin{tabular}{|c|c|c|c|c|}
\hline Profondeur (m) & $\begin{array}{c}\text { Contrainte } \\
\text { verticale } \\
\text { effective (MPa) } \\
\sigma^{\prime} v=\gamma^{\prime} . z\end{array}$ & $\begin{array}{l}\text { Contrainte } \\
\text { horizontale } \\
\text { effective (MPa) } \\
\sigma^{\prime} \mathrm{h}=\mathrm{K}_{0}, \sigma^{\prime} \mathrm{V}\end{array}$ & $\begin{array}{l}\text { Contrainte } \\
\text { venticale } \\
\text { totale (MPa) } \\
\sigma \mathrm{V}=\sigma^{\prime} \mathrm{V}+\mathrm{u}_{0}\end{array}$ & $\begin{array}{l}\text { Contrainte } \\
\text { horizontale } \\
\text { totale (MPa) } \\
\sigma h=\sigma^{\prime} h+t_{0}\end{array}$ \\
\hline $\begin{array}{c}\mathrm{z}=250 \mathrm{~m} \\
\gamma_{1}=19,91 \mathrm{kN} / \mathrm{m}_{3} \\
\mathrm{~K}_{0}=0,8\end{array}$ & 2,48 & 1,98 & 4,98 & 4,48 \\
\hline $\begin{array}{l}250 \mathrm{~m} \\
\gamma_{\mathrm{h}}=20,17 \mathrm{kN} / \mathrm{m}^{3} \\
\mathrm{~K}_{\mathrm{a}}=0,7\end{array}$ & 2,54 & 1.78 & 5.04 & 4,28 \\
\hline
\end{tabular}
que le massif reste élastique, permet de supporter la

TABLEAU Valeurs des contraintes in situ pour deux densités diffẻrentes.

TABLEAUII Composantes horizontales et verticales des contraintes de traction à appliquer sur les parois de la galerie.
$\beta=7.49$
$\beta=22,50$
$\beta=37.53$
$\beta=52.47$
$\beta=87.49$
$\beta=82,51$

\begin{tabular}{llllllll}
$\sigma_{n}(\mathrm{MPa})$ & 4,97 & 4,90 & 4,79 & 4,66 & 4,55 & 4,49 \\
\hline$\sigma_{\mathrm{nx}}(\mathrm{MPa})$ & 0,65 & 1,88 & 2,92 & 3,70 & 4,20 & 4,45 & \\
\hline$\sigma_{\mathrm{ny}}(\mathrm{MPa})$ & 4,93 & 4,53 & 3,80 & 2,84 & 1,74 & 0,58 & \\
\hline
\end{tabular}




\begin{tabular}{|c|c|c|c|c|c|c|}
\hline & $\sigma_{x}[\mathrm{MPa}]$ & $\begin{array}{l}\text { Elément } 1 \\
\sigma_{y}[\mathrm{MPa}]\end{array}$ & $\sigma_{1}[\mathrm{MPa}]$ & $\sigma_{x}[\mathrm{MPa}]$ & $\begin{array}{c}\text { Elément } 31 \\
\sigma_{y}[\mathrm{MPa}]\end{array}$ & $\sigma_{k}[\mathrm{MPa}]$ \\
\hline Cam-Clay & 3,21 & 1,04 & 2,66 & 0,82 & 3,09 & 2.54 \\
\hline $\begin{array}{l}\text { Modèle de } \\
\text { gonflement }\end{array}$ & 3,25 & 1,09 & 2,26 & 0,86 & 3,16 & 2,17 \\
\hline
\end{tabular}

décompression des contraintes radiales agissant loin du massif ( $($ effet de voûte »), qui diminuent lorsque le massif commence à se plastifier.

Les deux modèles (Cam-Clay et le modèle de gonflement) font apparaître une chute des contraintes dans la zone plastique à proximité de la paroi ; ces contraintes augmentent au fur et à mesure que l'on s'éloigne de celle-ci (zones moins perturbées par l'excavation). L'allure des courbes d'évolution des contraintes en fonction des incréments de décharge est pratiquement la même pour les deux modélisations. On présente uniquement les résultats du calcul avec le modèle de gonflement (Fig. 9a et 9b). Les contraintes finales obtenues avec les deux modélisations en clef de voûte et en piédroit sont données dans le tableau III.

Les chemins de contraintes locaux Q-P, obtenus par les deux modélisations, sont quasiment identiques pour le point de Gauss 121 (Fig. 8). En revanche, ils présentent une légère différence pour le point 1 avec des valeurs de déviateurs plus faibles pour le modèle de gonflement (Fig. 10a et 10b). La rupture du matériau est obtenue après une diminution de la pression moyenne et une augmentation du déviateur de contrainte.

Dans la modélisation du creusement d'une galerie circulaire, les résultats obtenus montrent que la diffé- rence entre les contraintes finales calculées avec les deux modèles (Cam-Clay et le modèle de gonflement) n'est pas très significative. Par conséquent, l'utilisation d'un modèle simple suffit pour évaluer les contraintes dues au creusement d'une galerie. En revanche, lors d'une décharge unidirectionnelle (cas des fouilles et des fondations), le calcul effectué avec les deux modèles montre un écart de $3 \mathrm{~cm}$ au niveau des déplacements en paroi, et un écart de $0,4 \mathrm{MPa}$ entre les contraintes. Pour de telles configurations, l'utilisation d'un modèle intégrant le phénomène de gonflement devient nécessaire pour dimensionner ce type d'ouvrages.

\section{b) Déplacements dans le massif}

On compare sur les figures 11 a et $11 \mathrm{~b}$ la convergence des nceuds 1 et 43 (nceuds représentés sur la figure 8). On remarque que la convergence prédite par le modèle de gonflement est supérieure à celle du modèle Cam-Clay. Les déplacements et les convergences finals de ces nceuds sont donnés dans le tableau IV.

La figure 12 donne l'évolution des déplacements obtenus suivant l'axe vertical avec les méthodes analytiques et numériques. Le calcul élastique sous-estime les déplacements : en effet le déplacement maximal
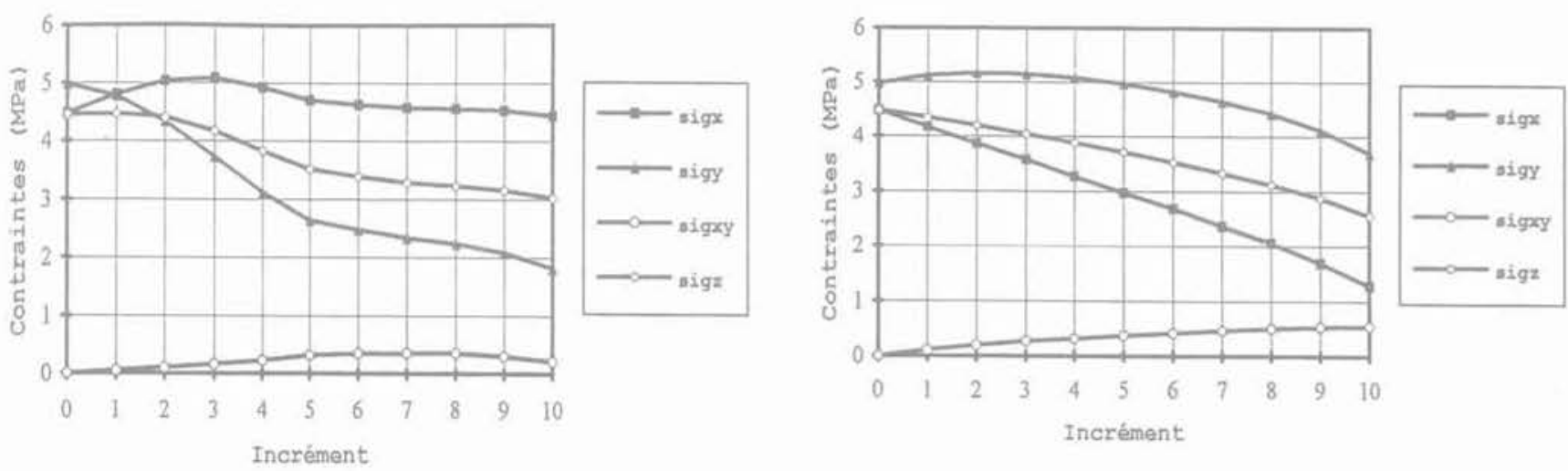

HG.9 Evolution des chemins de contraintes dans le massif pendant la décharge (a) Point 1. (b) Point 121. Evolution of stress paths in rock mass during unloading. (a) Point 1. (b) Point 121.

TABLEAU IV Déplacements et convergences finals obtenus avec les deux modèles.

\begin{tabular}{|c|c|c|c|c|}
\hline & & & & \\
\hline & Déplacement Uy $(\mathrm{cm})$ & Convergence Ui ( $\%)$ & Déplacement Ux (cm) & Convergence Ui $(\%)$ \\
\hline Cam-Clay & 8,01 & 2.67 & 7,81 & 2,60 \\
\hline $\begin{array}{l}\text { Modèle de } \\
\text { gonflement }\end{array}$ & 9,50 & 3,17 & 8,28 & 2,76 \\
\hline
\end{tabular}



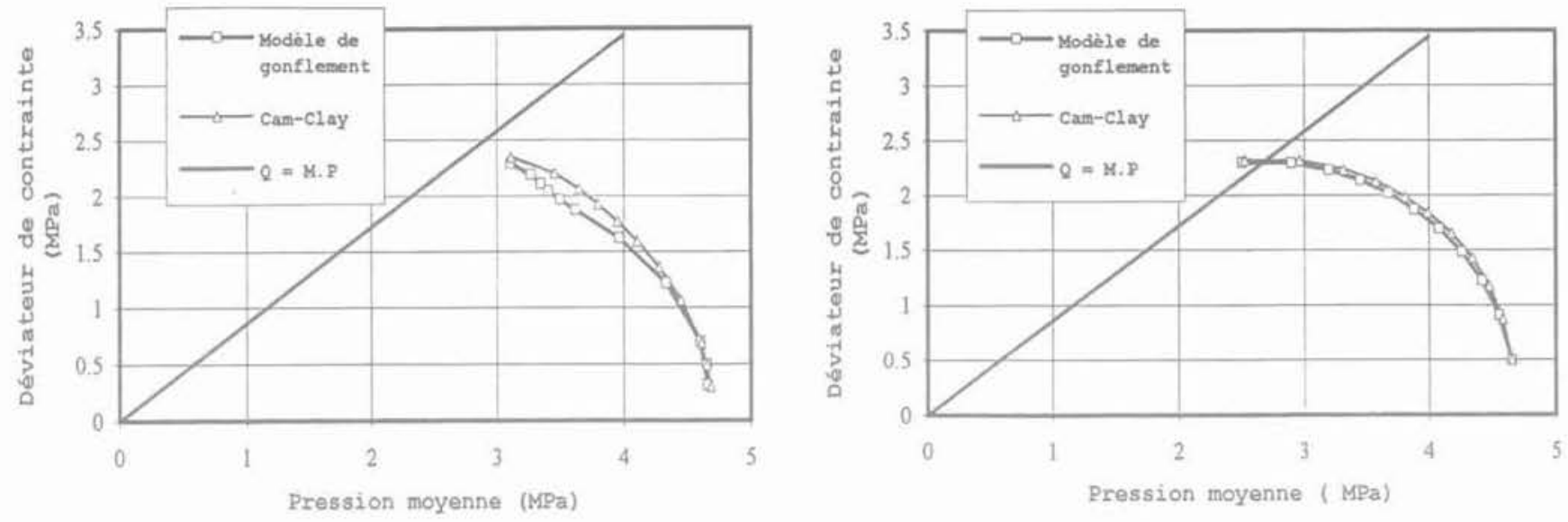

HQ. 10 Évolution des chemins de contraintes locales pendant la décharge - comparaison des deux modélisations. (a) Point 1. (b) Point 121.

Evolution of local stress paths during unloading - comparison between modelling. (a) Point 1. (b) Point 121.
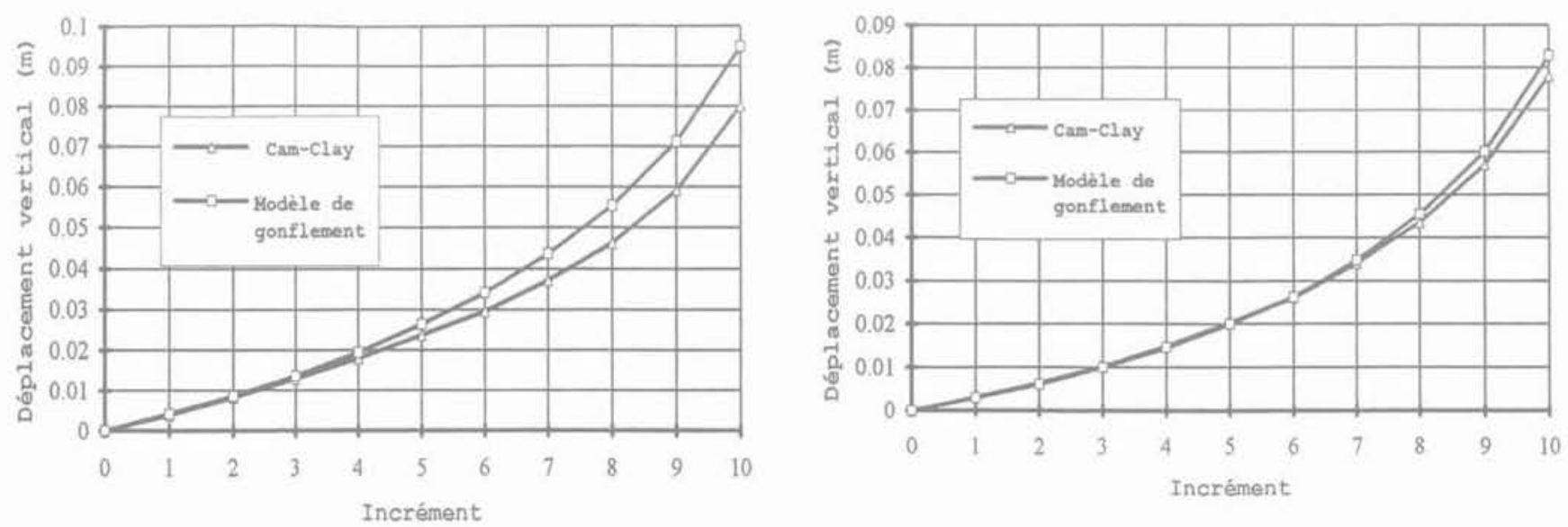

FG. 11 Évolution des déplacements au cours

de la décharge - comparaison des deux modélisations.

(a) Nœud 1. (b) Nœud 43.

Evolution of displacements during unloading - comparison between modelling.

(a) Node 1. (b) Node 43.

obtenu en paroi est de $4 \mathrm{~cm}$. Les résultats du critère de Mohr-Coulomb montrent que des déplacements importants ont lieu au niveau de la zone plastique proche de la paroi, pour ensuite diminuer brusquement dans la zone élastique, le déplacement maximal est de $6,7 \mathrm{~cm}$. L'utilisation des modèles numériques avec écrouissage conduit à une convergence plus forte (Tableau IV) et plus régulière, ce qui est plus représentatif du comportement réel du massif pendant la décharge.

La convergence de la galerie ainsi que les zones entrées en plasticité obtenues avec les deux modélisations numériques sont représentées sur les (Fig. 13a et $13 \mathrm{~b})$ et montrent que la déformée de la galerie reste circulaire. La convergence et l'étendue de la zone plastique sont plus importants dans le cas du modèle de gonflement (car le nombre de points de Gauss entrés en plasticité est plus élevé).

\section{6}

\section{Conclusion}

La modélisation du comportement à long terme d'un ouvrage souterrain installé dans une argile gon- flante a été illustrée à travers un modèle élastoplastique couvrant seulement les aspects contraintes et déformations sans couplage avec le fluide interstitiel. Le modèle élastoplastique utilisé est à deux mécanismes plastiques, dont l'un est associé aux contraintes de contact

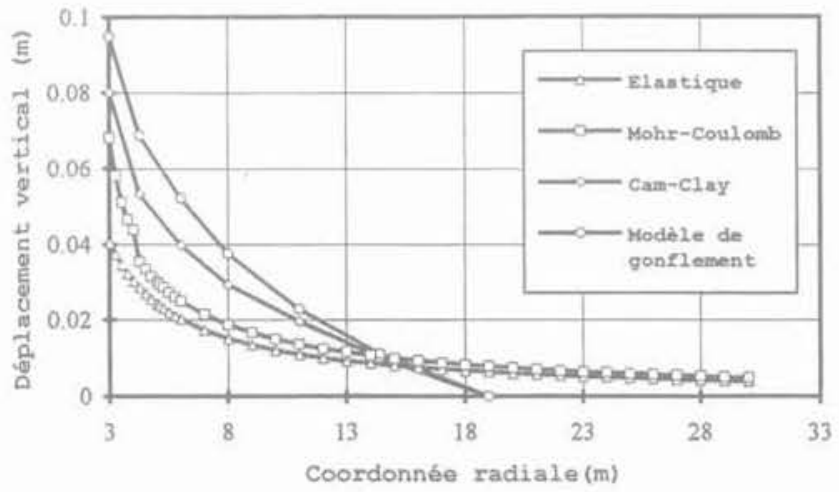

FiG. 12 Déplacements suivant $l^{\prime}$ axe vertical prédit par les différentes approches analytiques et numériques.

Displacements along the vertical axe predicted by several approaches, analytical and numerical. 


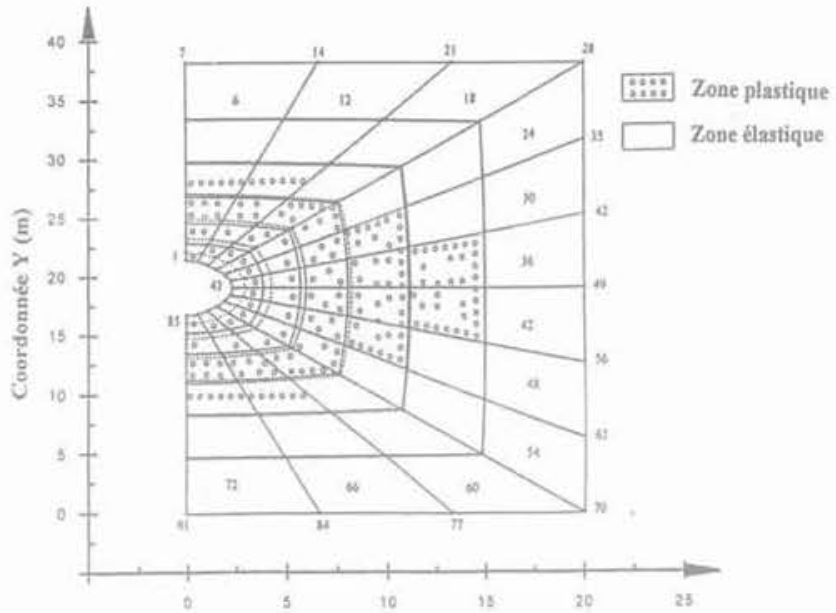

f16. 13 Déformée de la galerie et étendue des zones plastiques obtenues au dixième incrément.

(a) Calcul avec le modèle Cam-Clay. (b)

Calcul avec le modèle de gonflement.

Deformation of gallery and plastic zone

extension obtained after the tenth increment.

(a) Calculation with Cam-Clay model.

(b) Calculation with swelling model.

par la surface de charge « $\mathrm{f})$; le second mécanisme traduit les contraintes de répulsion par la surface de charge «F », Ce modèle permet de tenir compte du gonflement élastoplastique observé en décharge sur des argiles saturées gonflantes. Le modèle comporte huit paramètres et a été introduit dans un code de calcul par éléments finis. La validation du code a été réalisée sur des essais homogènes de laboratoire (essais odométriques et triaxiaux), et montre que le comportement est correctement traduit par cette modélisation.

L'application du modèle à la simulation du creusement d'une galerie circulaire installée dans l'argile de Boom (gonflante) a permis de retenir les constatations suivantes :

- les résultats du calcul montrent une faible ovalisation de la section de la galerie (le déplacement en clef de voûte est légèrement supérieur à celui en piédroit) : - la convergence maximale de la galerie obtenue par le calcul est observée en clef de voûte, et elle est de l'ordre

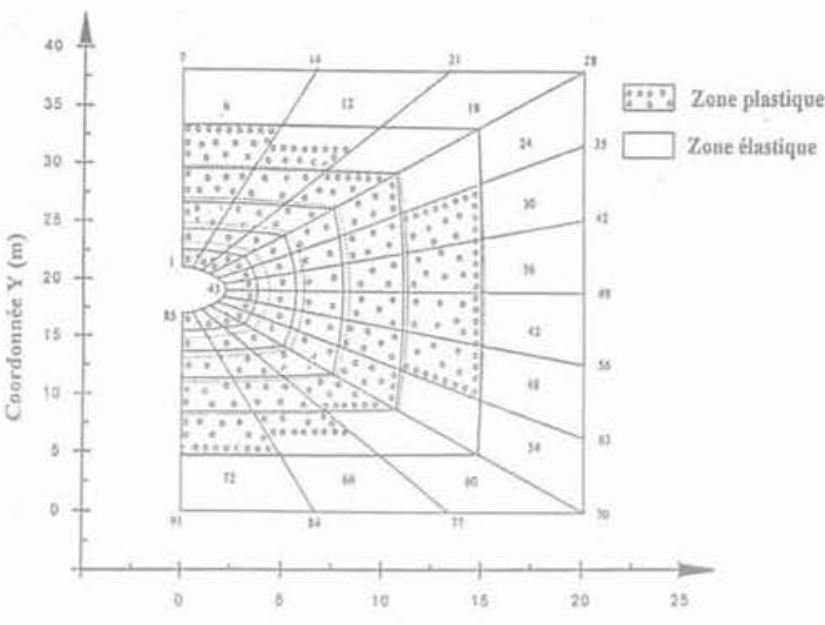

de $2,67 \%$ pour le modèle Cam-Clay et de $3,17 \%$ pour le modèle de gonflement. Ces convergences sont comparables à celles obtenues par un calcul similaire avec un modèle élasto-visco-plastique effectué par Rousset (1988), qui donne une convergence à la paroi de la galerie avant la pose du soutènement de $3,49 \%$;

- les valeurs de contraintes finales sont bien plus faibles que celles attendues dans une situation géostatique. La valeur de la contrainte verticale est comprise entre 2,6 et $3,2 \mathrm{MPa}$, et la valeur de la contrainte horizontale est comprise entre 4,1 et $4,5 \mathrm{MPa}$ à une profondeur de 12 à $15 \mathrm{~m}$. Les mesures de contraintes, effectuées sur le site de Mol en Belgique avec l'appareil autoforeur dans une galerie cintrée, proposent des valeurs de contraintes verticales comprises principalement entre 3,5 et $4 \mathrm{MPa}$ à une distance de 12 à $15 \mathrm{~m}$ de la galerie (Bonne et al., 1993). Les contraintes calculées sont proches des contraintes mesurées et le calcul montre que le comportement de la galerie en décharge est correctement décrit.

\section{Bibliographie}

Baldi G., Hueckel T., Peano A., Pellegrini R. - Development in modelling of the thermo-hydro-geomechanical behaviour of Boom clay and clay based buffer materials. Final report for CEC (F11W/0150 a), ISMES, Bergamo, Italy, 1990.

Bernaud D., Rousset G. - Dimensionnement du soutènement des galeries creusées dans une argile profonde contrat $n^{2}$ F11W/0112, Sciences et techniques nucléaires, Paris, 1991

Börgesson I., Karnland O., Johannesson L.E. - Modelling of physical behaviour of clay barriers close to water saturation. Clay Technology AB, Lund, Sweden, 1993
Chen F.H. - Foundations on expansive soils. N.Y., Elsevier, 1975.

Chen F.H. - Foundations on expansive solls. Amsterdam, Elsevier, Developments in Geotechnical Engineering. vol. 54, 1988.

Delville A. - Modelling the clay water interface. Langmuir 7, 1991, p. 547-555.

Didier G., Soyez B., Héritier B, Parez L. Étude à l'oedomètre du gonflement des sols. Congrès International sur les sols gonflants, Dublin, 1987

Giraud A.-Couplage thermo-hydromécanique dans les milieux poreux peu perméables : application aux argiles profondes. These de Doctorat, ENPC, Paris, 1993.
Israelachvili S.N. - Intermolecular and surface forces. Acad. press, Orlando, FA, 1985.

Israelachvili S.N. - Intermolecular and surface forces (Second edition). San Diego, Acad press, 1991.

Lambe T.W. - The engineering behaviour of compacted clay, Journal of the soil mech and found. ASCE div., paper 1655, 1958, p. 1-35.

Mitchell J.K - Fundamentals of soil behaviour. John Wiley, New York, 1976.

Mohan D. - Pile foundations. Rotterdam, A.A. Balkema, 1988

Mohan D., Chandra S. - Frictional resistance of bored piles in expansive clays. Geotechnique, vol. 11, 4, 1961, p. 294 301. 
Mouroux P., Margron P., Pinte J.-C. - La construction économique sur sols gonflants. BRGM, Manuels et méthodes 14 1988.

Pakzad M. - Modélisation du comportement hydro-mécanique des argiles gonflantes saturées et partiellement saturées à faible porosité. Thèse de Doctorat, Université d'Orléans, 1995

Picard J.-M., Rousset G. - Essai hydrothermo-mécanique dans une argile profonde (Mol, Belgique), essai «CACTUS W is, Contrat FI2W/0001F(CD), Commission des communautés européennes, sept. 1993.
Prakash C., Narayan S., Chandra R., Balhodi G.R., Price G., Wardle I.F. - Pile instrumentation in expansive soils. Proc. 6th International Conference on Expansive Soils, New Delhi, vol. 1, 1987, p. 289-294.

Robinet J.-C. Pakzad M. Plas F. - Un modèle rhéologique pour les argiles gonflantes, Revue Française de Géotechnique $n^{\circ}$ 68, $3^{\circ}$ trimestre, 1994.

Rousset G. - Comportement mècanique des arailes profondes application au stockage de déchets radioactifs. Thèse de Doctorat, ENPC, Paris, 1988.

Sorochan E.A. - Construction d'ouvrages sur les sols gonflants. Moscou, 1989.
Sridharan A. - Strength and volume change behaviour of a sand bentonite mixture. Can Geotech. journal 27, 404. 1990.

Tacherifet S. - Modélisation du comportement mécanique des argiles gonflantes application aux ouvrages de stockage. Thèse de Doctorat, Université d'Orléans. 1995.

Van Damme H., Bergaya F., Gatineau L.Contraintes structurales sur la réactivité dans les argiles et les solides très divisés. Journal de chimie physique $84, \mathrm{n}^{\circ} 9$ 1987 , p. 1075-1082

\section{Annexe I Formulation du modèle}

Le modèle comporte deux parties.

- Pour les états de contraintes normalement consolidés, le modèle de calcul est celui de Cam-Clay modifié.

La surface de charge est donnée par

$$
f(\sigma)=q^{2}-M^{2} p\left(2 p_{d}-p\right)=0
$$

avec:

$$
M=\frac{6 \sin (\phi)}{3-\sin (\phi)} ; P_{c f}=p_{c 0} \exp \left(\beta_{1} \varepsilon_{v}^{p}\right) ; \beta_{1}=\frac{1+e_{0}}{\gamma_{1}-k}
$$

avec pour notations :

$\mathrm{M}$ : paramètre d'état critique correspondant à un état de contrainte pour lequel les déformations volumiques plastiques sont nulles;

$\mathrm{P}_{c 0}$ : pression de préconsolidation;

$\mathrm{p}_{\mathrm{f}}$ : pression de consolidation qui désigne le paramètre d'écrouissage:

$\varepsilon_{\mathrm{v}}$ : déformation volumique plastique ;

$\beta_{1}$ : module de compressibilité plastique:

$\lambda_{1}$ et $\kappa$ : pentes élastoplastique et élastique du matériau, déterminées à partir d'essais isotropes ou odométriques;

$e_{0}$ : indice des vides initial du matériau déterminé au laboratoire.

Les déformations plastiques sont données par:

$$
d \varepsilon_{i j}^{p}=\lambda \frac{\partial f}{\partial \sigma_{i j}} ; d \varepsilon_{v}^{p}=\lambda \frac{\partial f}{\partial p} ; d \varepsilon_{q}^{p}=\lambda \frac{\partial f}{\partial q}
$$

$\lambda$ est le multiplicateur plastique donné par la condition de consistance $(\mathrm{df}=0)$ :

$$
\lambda=\frac{\frac{\partial f}{\partial \sigma}[C] d \varepsilon^{t o t}}{\frac{\partial f}{\partial \sigma}[C] \frac{\partial f}{\partial \sigma}-\frac{\partial f}{\partial \varepsilon_{v}^{p}} \frac{\partial f}{\partial p}}
$$

avec:

[C], matrice d'élasticité;

do, vecteur incrément de contraintes effectives $\mathrm{d} \varepsilon^{t 0 t}$, vecteur incrément de déformations totales.

- Pour les états de contraintes surconsolidés, la deuxième surface, dite de gonflement est introduite: elle est en contact avec celle de Cam-Clay au point de déchargement. Cette dernière permet de calculer les déformations plastiques engendrées par le gonflement pendant la décharge.

L'expression de cette surface est donnée par;

$$
F=\left(q-\alpha_{q}\right)^{2}+M^{2} \cdot\left(p-\alpha_{p}+a_{1}\right)\left(p-\alpha_{p}-a_{p}\right)=0
$$

$\alpha_{\mathrm{v}}$ et $\alpha_{c}$ sont les coordonnées du centre de l'ellipse, définies par les équations ci-dessous. Elles sont calculées en fonction des contraintes initiales de décharge $\left(p_{1}, q_{1}\right)$, et de l'état actuel des contraintes $(p, q)$, soit:

$$
\alpha_{p}=\frac{P+P_{1}}{2} ; \alpha_{q}=\frac{q+q_{1}}{2}
$$

a est le paramètre d'écrouissage isotrope (grand rayon de la surface de gonflement).

L'évolution du paramètre d'écrouissage a est donnée par:

$$
\mathrm{da}_{1}=\mathrm{B}_{1} \mathrm{~d} \varepsilon_{\mathrm{p}}^{\mathrm{n}}+\mathrm{B}_{2} \mathrm{~d} \varepsilon_{\mathrm{q}}^{\mathrm{p}}
$$

$\mathrm{da} \mathrm{a}_{1}=\mathrm{B}_{1} \cdot \mathrm{d} \varepsilon_{\mathrm{v}}^{\mathrm{p}}+\mathrm{B}_{2} \cdot \mathrm{d} \varepsilon_{\mathrm{q}}^{\mathrm{p}}$

avec:

$$
B_{1}=p \beta_{2} \frac{\partial F / \partial p}{-2 \partial F / \partial a_{1}} ; B_{2}=M^{2} p \beta_{2} \frac{\partial F / \partial q}{-2 \partial F / \partial a_{1}}
$$

$\beta$, représente le module de compressibilité plastique à l'état surconsolidé, il est donné par:

$$
\left(\beta_{2}=\frac{1+e_{0}}{\lambda_{2}-K}\right)
$$

- en décharge: $\beta_{2}=\beta_{d}=\beta_{1}\left(1+\frac{\left(p_{c}\right) \text { ref }}{\left(P_{c}\right) \text { dech }}\right) \frac{P_{c}}{p}$

- en recharge : $\beta_{2}=\beta_{r}=\beta_{1}\left(1+\frac{\left(p_{c}\right) \text { ref }}{p_{c}}\right) \frac{2 p_{c t}}{3\left(P_{c}\right)_{\text {dech }}} \frac{P}{P_{c}}$

Notons que:

(p) $)_{\text {fuf }}$ est une pression de consolidation de référence à partir de laquelle on effectue une décharge dans un essai oedométrique. A partir de cette pression il est possible de déterminer le module de compressibilité $\beta$. pour n'importe quelle pression de décharge;

$\left(\mathrm{p}_{\mathrm{c}}\right)_{\text {dect }}$ est une pression de consolidation à partir de laquelle on effectue la décharge; 
$p_{c}$ est une pression de consolidation fictive correspondant à l'état de contrainte actuel.

Les déformations volumiques et déviatoires plastiques sont données par:

$$
\mathrm{d} \varepsilon_{\mathrm{v}}^{\mathrm{p}}=\lambda \frac{\partial \mathrm{F}}{\partial \mathrm{p}} \mathrm{d} \varepsilon_{\mathrm{q}}^{p}=\lambda\left(\frac{\partial \mathrm{F}}{\partial \mathrm{q}}-\alpha \frac{\partial \mathrm{F}}{\partial \mathrm{p}}\right)
$$

avec

$$
\alpha=\frac{P_{c}-0,5\left(P_{c}\right)_{\text {dech }}}{M^{2}\left(P_{c}\right)_{\text {dech }}}
$$

$\alpha$ est un paramètre introduit pour exprimer une relation non linéaire entre dq et dp. Le multiplicateur plastique est donné par la condition de consistance $(\mathrm{dF}=0)$ :

$$
\lambda=\frac{\frac{\partial F}{\partial \sigma} C d \varepsilon^{t o t}}{\frac{\partial F}{\partial p}\left(K+\beta_{2}\right) \frac{\partial F}{\partial p}+\frac{\partial F}{\partial q}\left(3 G+M^{2} p \beta_{2}\right)\left(\frac{\partial F}{\partial q}-\alpha \frac{\partial F}{\partial p}\right)}
$$

\section{Annexe II \\ Formulation variationnelle du problème mécanique}

1. Équations du problème mécanique à résoudre Équation d'équilibre

$$
\sigma_{i j, j}+f_{i}=0
$$

Le modèle de comportement

$$
\Delta \sigma_{u}=C_{i j k} \cdot \Delta \varepsilon_{k j}-\Delta \sigma_{i j}^{0}
$$

avec:

$$
\varepsilon_{i j}=\frac{1}{2}\left(u_{i j}+u_{j i}\right)
$$

\section{Conditions aux limites}

Le domaine a de frontière r est soumis aux sollicitations suivantes:

- forces volumiques $f_{\text {, dans }} \mathrm{n}$;

- forces de surfaces $T_{1}=\bar{T}_{1}=\sigma \cdot n$ sur $r_{\sigma^{*}}$;

- déplacement imposé $u_{4}=\bar{u}_{i}$ sur $r_{u}$

\section{Formulation variationnelle du problème}

On considère l'espace $V$ des champs de déplacements cinématiquement admissibles.

$$
\mathrm{V}=(\mathrm{v} / \mathrm{v} \text { régulier sur a et } \mathrm{v}=0 \text { sur ru })
$$

Le principe des travaux virtuels s'écrit :

$$
-\int_{\Omega} \varepsilon_{i j}(v) \sigma_{i j}(u) d \Omega+\int_{\Omega} f_{i} v_{i} d \Omega+\int_{\Gamma_{0}} \bar{T}_{i} v_{i} d \Gamma=0
$$

\section{Discrétisation de l'espace}

a) Approximation du champ de déplacement

$$
\mathrm{v}^{\mathrm{e}}=\mathrm{N}^{\mathrm{e}} \cdot \mathrm{u}^{\mathrm{e}}
$$

$\mathrm{u}^{\mathrm{e}}$ : déplacement aux nœuds de l'élément de référence ;

$\mathrm{N}^{\mathrm{e}}$ : matrice des fonctions d'interpolations.

b) Approximation du champ de déformation

$$
\varepsilon=\mathrm{L} \cdot \mathrm{U}=\mathrm{L} \cdot \mathrm{N} \cdot \mathrm{u}^{\mathrm{e}}=\mathrm{B} \cdot \mathrm{u}^{\mathrm{e}}
$$

L'équation (4) peut s'écrire sous la forme:

$$
\begin{gathered}
\int_{\Omega} \varepsilon^{t} \sigma d \Omega-\int_{\Omega} f v^{t} d \Omega-\int_{r_{\sigma}} T v^{t} d \Gamma=0 \\
\int_{\Omega} u^{t} B \sigma d \Omega-\int_{\Omega} f(N u)^{t} d \Omega-\int_{\Gamma_{\sigma}} T(N u)^{t} d \Gamma=0
\end{gathered}
$$

soit:

$$
\begin{gathered}
u^{t}\left[\int_{\Omega} B^{t} \sigma d \Omega-\int_{\Omega} N^{t} f d \Omega-\int_{\Gamma_{\sigma}} N^{t} T d \Gamma\right]=0 \\
{\left[\int_{\Omega} B^{t} \sigma d \Omega-\int_{\Omega} N^{t} f d \Omega-\int_{\Gamma_{\sigma}} N^{t} T d \Gamma\right]=0}
\end{gathered}
$$

Pour l'incrément $k$, les sollicitations appliquées à a peuvent s'écrire:

$\mathrm{f}^{(\mathrm{k})}=\mathrm{f}^{(\mathrm{k}-1)}+\Delta \mathrm{f}^{\mathrm{k})}$ dans $\mathrm{a}$

$\overline{\mathrm{u}}^{(\mathrm{k})}=\overline{\mathrm{u}}^{(\mathrm{k}-1)}+\Delta \overline{\mathrm{u}}^{(\mathrm{k})}$ sur $\mathrm{r}_{\mathrm{u}}^{\mathrm{k}}$

$T^{k+}=T^{k-1 k}+\Delta T^{k k}$ sur $r_{a}^{k}$

en outre les déplacements, les contraintes et les déformations à la fin du $\mathrm{k}^{\mathrm{e}}$ incrément s'écrivent:

$$
\begin{gathered}
u^{(k)}=u^{(k-1)}+\Delta u^{i k)}, \sigma^{(k)}=\sigma^{(k-1)}+\Delta \sigma^{(0)} \\
\varepsilon^{(k)}=\varepsilon^{(k-1)}+\Delta \varepsilon^{(k)}
\end{gathered}
$$

Ainsi l'équation (10) s'écrit :

$$
\int_{\Omega} B^{t} \Delta \sigma^{k} d \Omega-\int_{\Omega} N^{t} \Delta f^{k} d \Omega-\int_{\Gamma_{0}} N^{t} \Delta T^{K} d \Gamma+F R=0
$$

avec:

$$
F R=\int_{\Omega} B^{T} \sigma^{k-1} d \Omega-\int_{\Omega} N^{T} f^{k-1} d \Omega-\int_{T_{0}} N^{t} T^{k-1} d \Gamma
$$

FR: représente les forces non équilibrées des incréments précédents.

En tenant compte de la loi de comportement non linéaire du matériau :

d'après (11)

$$
\Delta \sigma^{\mathrm{k}}=\mathrm{C} \cdot \Delta \varepsilon^{\mathrm{k}}=\mathrm{C} \cdot \mathrm{B} \cdot \Delta \mathrm{u}^{\mathrm{k}}
$$

$$
\int_{\Omega} B^{i} C B \Delta u^{k} d \Omega=\Delta F
$$

avec:

$$
K=\int_{\Omega} B^{t} C B d \Omega
$$

K définit la matrice de rigidité globale de la structure; $\Delta \mathrm{U}$ est un vecteur représentant l'accroissement des déplacements nodaux;

$\Delta \mathrm{F}$ représente l'accroissement des forces extérieures.

\section{Résumé du problème discrétisé posé}

Le problème ainsi posé se résume dans les deux systèmes suivants :

- un système global

$$
\mathrm{K} . \Delta \mathrm{U}=\Delta \mathrm{F}
$$

- des systèmes locaux

$$
\Delta \sigma=C . \Delta \varepsilon
$$

